\title{
Effect of Electron-Phonon Interaction Range for a Half-Filled Band in One Dimension
}

\author{
Martin Hohenadler, ${ }^{1}$ Fakher F. Assaad, ${ }^{1}$ and Holger Fehske ${ }^{2}$ \\ ${ }^{1}$ Institut für Theoretische Physik und Astrophysik, Universität Würzburg, 97074 Würzburg, Germany \\ ${ }^{2}$ Institut für Physik, Ernst-Moritz-Arndt-Universität Greifswald, 17487 Greifswald, Germany
}

(Dated: March 7, 2022)

\begin{abstract}
We demonstrate that fermion-boson models with nonlocal interactions can be simulated at finite band filling with the continuous-time quantum Monte Carlo method. We apply this method to explore the influence of the electron-phonon interaction range for a half-filled band in one dimension, covering the full range from the Holstein to the Fröhlich regime. The phase diagram contains metallic, Peierls, and phase-separated regions. Nonlocal interactions suppress the Peierls instability, and thereby lead to almost degenerate power-law exponents for charge and pairing correlations.
\end{abstract}

PACS numbers: 71.10.Hf, 71.10.Pm, 71.30.+h, 71.45.Lr

Introduction.-Electron-phonon interaction has an essential influence on the properties of many materials [1]. It plays a key role for pairing and superconductivity, mass renormalization, and charge ordering phenomena. Taking into account quantum lattice fluctuations leads to a complex, many-body problem. Consequently, theoretical studies usually rely on simplified microscopic models. A frequently invoked approximation, in particular for numerical studies, is to consider a completely local electronphonon coupling as in Holstein's molecular-crystal model [2]. However, nonlocal interactions are expected to play an important role in materials with incomplete screening such as quasi-one-dimensional (quasi-1D) organics 3. Long-range interactions, as described by the Fröhlich model [4, have been investigated in the context of hightemperature superconducting cuprates [5] 2], and were found to support light polarons and bipolarons [10 12.

Exact numerical methods have played an important role for the understanding of coupled electron-phonon systems. Whereas efficient algorithms exist for Holsteintype models at arbitrary band filling [13 18, extended interactions could so far be addressed only in the emptyband limit, see, for example, $10,19 \sqrt{22}$, and 8 for a review. Consequently, key phenomena such as the Peierls instability 23] were out of reach. The latter occurs in quasi-1D systems with commensurate fillings, for example in TTF-TCNQ 24, and drives a transition to a Peierls insulator with charge-density-wave order. For the Holstein model, it is known that quantum lattice fluctuations can destroy the charge order [25], leading to a metal-insulator quantum phase transition at a finite value of the electron-phonon coupling strength.

Unbiased investigations of the effect of nonlocal interactions at finite band-filling represent a long-standing, open problem. In this Letter, we use the continuoustime quantum Monte Carlo (CTQMC) method [26] to study a model that interpolates between and includes the paradigmatic Holstein and Fröhlich limits.

Model.-We consider a Hamiltonian $\hat{H}=\hat{H}_{0}+\hat{H}_{1}$, where $\hat{H}_{0}=-t \sum_{\langle i j\rangle \sigma}\left(c_{i \sigma}^{\dagger} c_{j \sigma}+\right.$ H.c. $)$ describes $1 \mathrm{D}$ fermions with nearest-neighbor hopping $t$, and

$$
\hat{H}_{1}=\sum_{i}\left(\frac{1}{2 M} \hat{P}_{i}^{2}+\frac{K}{2} \hat{Q}_{i}^{2}\right)-\gamma \sum_{i, r} f(r) \hat{Q}_{i+r}\left(\hat{n}_{i}-1\right) .
$$

The first term describes lattice fluctuations in the harmonic approximation, with the phonon frequency $\omega_{0}$ and the stiffness constant $K=\omega_{0}^{2} M$. The second term represents the electron-phonon interaction, in the form of a nonlocal density-displacement coupling, with the density operator $\hat{n}_{i}=\sum_{\sigma} \hat{n}_{i \sigma}$ and $\hat{n}_{i \sigma}=c_{i \sigma}^{\dagger} c_{i \sigma}$. The matrix elements are chosen as [9, 10, 27.

$$
f(r)=\frac{1}{\left(r^{2}+1\right)^{3 / 2}} e^{-r / \xi}, \quad 0 \leq r<L / 2,
$$

where the lattice constant $a=1$. For $\xi \rightarrow \infty$, this coupling represents a lattice version of the Fröhlich interaction 27. More generally, $\hat{H}_{1}$ may be viewed as an extended Holstein interaction with screening length $\xi$; the original Holstein model [2] is recovered in the limit $\xi \rightarrow 0$. For $\omega_{0} \rightarrow \infty$, the model maps onto an attractive, generalized Hubbard model. Our method can be applied to any coupling which preserves translational invariance. The restriction of $r$ is due to periodic boundary conditions.

Method.-For electron-phonon problems [18], the starting point is the partition function at inverse temperature $\beta=1 / k_{\mathrm{B}} T$,

$$
Z=\int \mathcal{D}(\bar{\psi}, \psi) e^{-S_{0}[\bar{\psi}, \psi]} \int \mathcal{D}(q) e^{-S_{1}[\bar{\psi}, \psi, q]},
$$

where $\bar{\psi}, \psi$ are Grassmann fields, and $q$ denotes phonon coordinates. The phonons can be integrated out exactly 28, leading to a purely fermionic action with a nonlocal (in space and time) interaction

$$
S_{1}^{\mathrm{f}}=-\iint_{0}^{\beta} d \tau d \tau^{\prime} \sum_{i j}\left[n_{i}(\tau)-1\right] D_{i, j}^{\tau, \tau^{\prime}}\left[n_{j}\left(\tau^{\prime}\right)-1\right] .
$$

The phonon propagator takes the form $D_{i, j}^{\tau, \tau^{\prime}}=F(i-$ j) $D\left(\tau-\tau^{\prime}\right)$ with $F(i-j)=\sum_{r} f(r+i-j) f(r)$ and 
the Holstein propagator $D\left(\tau-\tau^{\prime}\right)$. The interaction range in space (time) is determined by $\xi\left(\omega_{0}\right)$. The CTQMC method used here is based on an exact expansion around $\gamma=0$ [26]. A hybridization expansion algorithm for electron-phonon impurity problems also exists [29]. Monte Carlo updates consist of adding or removing single vertices, and flipping auxiliary Ising spins [18, 26]. The numerical effort scales with the cube of the average expansion order. Because of the underlying weakcoupling expansion, the CTQMC method is particularly efficient for problems with small expansion orders, such as the Peierls transition in the adiabatic regime $\omega_{0} / t \ll 1$ [30. Importantly, the method enables us to study the many-electron problem [31. We have verified that it reproduces exact results in the Holstein limit $\xi \rightarrow 0$, and in the anti-adiabatic limit $\omega_{0} \rightarrow \infty$ where the model (1) maps onto an extended attractive Hubbard model.

Results.-We choose a phonon frequency $\omega_{0} / t=0.5$. In this regime, representative of many materials, neither static nor instantaneous approximations are valid, and numerical simulations are essential. The dimensionless ratio $\lambda=\epsilon_{\mathrm{p}} / 2 t$, where $\epsilon_{\mathrm{p}}$ is the polaron binding energy in the atomic limit $(t=0)$ [27] and $2 t$ is half of the free bandwidth, allows us to compare different $\xi$ at the same effective coupling strength. We use $t$ as the energy unit, and set $\hbar=M=1$. All results are for a half-filled band.

The phase diagram as a function of $\xi$ and $\lambda$, obtained from CTQMC simulations with up to $L=42$ sites, is shown in Fig. 1. Because the Holstein model is recovered for $\xi=0$, its previously studied metallic and Peierls insulating phases [15, 32, 33] smoothly extend to $\xi>0$. However, we observe a significant $\xi$-dependence for small $\xi$, and saturation for larger values. Additionally, for sufficiently large $\xi$ and $\lambda$, we find a region of phase separation or charge segregation. The metallic phase and the metal-insulator transition extend all the way to the Fröhlich limit $\xi=\infty\left[\lambda_{\mathrm{c}}^{\mathrm{PI}}=0.48(2)\right]$; see also Fig. 3 . For the range of $\lambda$ shown in Fig. 1, phase separation is absent for $\xi \leq 2$, and becomes more favorable with increasing $\xi$.

The different phases can be characterized by the density correlator $S_{\rho}(r)=\left\langle\left(\hat{n}_{r}-1\right)\left(\hat{n}_{0}-1\right)\right\rangle$ and the density structure factor $S_{\rho}(q)$. In the metallic phase [Fig. 2(a)], $S_{\rho}(r)$ shows a power-law decay of $2 k_{\mathrm{F}}$ correlations (with exponent $K_{\rho}$ ) as expected from bosonization, and is linear for $q \rightarrow 0$. Together with exponentially suppressed spin correlations (not shown), these findings are consistent with a bipolaronic Luther-Emery phase [34, 35].

The Peierls state exhibits quasi-long-range $2 k_{\mathrm{F}}$ density correlations [Fig. 2(a)], corresponding to charge-densitywave order at $T=0$ with two electrons of opposite spin forming bipolarons on every other site. The phase boundary for the Peierls transition can be determined from the staggered charge susceptibility [32]

$$
\chi_{\rho}(\pi)=\frac{1}{L} \sum_{i j}(-1)^{i-j} \int_{0}^{\beta} d \tau\left\langle\hat{n}_{i}(\tau) \hat{n}_{j}(0)\right\rangle .
$$

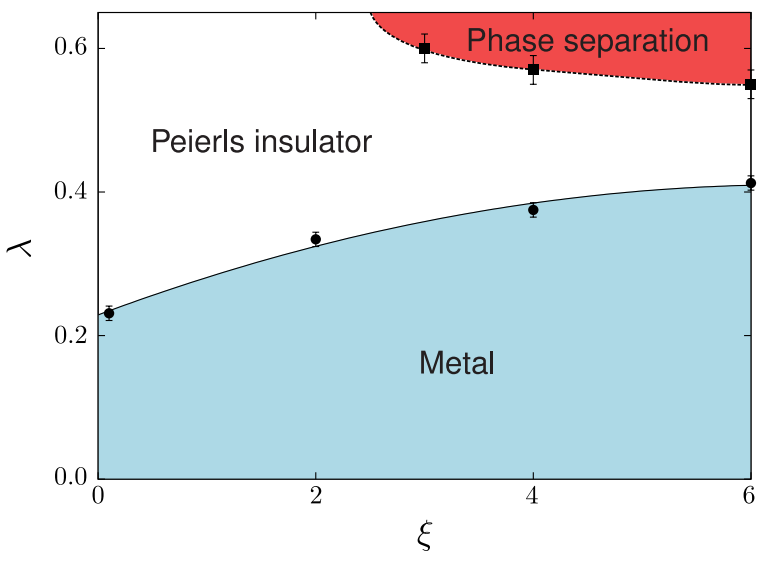

FIG. 1. (Color online) Phase diagram as a function of interaction range $\xi$ and electron-phonon coupling strength $\lambda$. The regions correspond to a metal, a Peierls insulator, and phase separation. The metallic and Peierls phases (and presumably also phase separation) extend to the Fröhlich limit $\xi=\infty$. Lines are guides to the eye. Here $\omega_{0} / t=0.5$.

For fixed $\beta / L, \chi_{\rho}(\pi) / L$ is universal at the critical point, and the crossing of curves for different $L$ gives $\lambda_{\mathrm{c}}^{\mathrm{PI}}$; for example, we have $\lambda_{\mathrm{c}}^{\mathrm{PI}}=0.33(1)$ for $\xi=2$ in Fig. 2(c). As for the Holstein model [32, 36], the Peierls transition is expected to be of the Kosterlitz-Thouless type also for $\xi>0$. Extended interactions $(\xi>0)$ promote metallic behavior by dissociating the onsite bipolarons predominant in the Holstein regime, and we see a Peierls insulator to metal transition as a function of $\xi$ [Fig. 2(a)]. By the same mechanism, the critical coupling for the transition to the Peierls insulator, $\lambda_{\mathrm{c}}^{\mathrm{PI}}$, increases with increasing $\xi$, see Fig. 1. The onset of charge order is also reflected in the divergent $q=2 k_{\mathrm{F}}$ peak in $S_{\rho}(q)$, see Fig. 2(b).

Phase separation as a result of the phonon-induced attraction manifests itself as a peak at small $q$ in $S_{\rho}(q)$, as shown in Fig. 2(b). In the phase-separated region of Fig. 1. the quantity $\pi S_{\rho}\left(q_{1}\right) / q_{1}$ (with $q_{1}=2 \pi / L$ )-whose thermodynamic limit is related to $K_{\rho}$ in a Luttinger liquid - diverges with system size. In the Peierls phase $K_{\rho}=0$, as verified on very large systems 32. Formally, $K_{\rho}=\infty$, reflecting phase separation, implies a divergent compressibility 34. The divergence of $\pi S_{\rho}\left(q_{1}\right) / q_{1}$ in the phase-separated region is shown for $\xi=6$ in Fig. 2(d), and we deduce a critical value of $\lambda_{\mathrm{c}}^{\mathrm{PS}}=0.55(2)$. There are two possible scenarios for the transition from the Peierls to the phase separated region. A continuous transition would imply a melting of the charge-ordered Peierls state before the formation of multipolaron droplets, allowing for an intervening (narrow) metallic region with finite $K_{\rho}$. Alternatively, the insulator-insulator transition could be of first order. Evidence for the latter possibility comes from the occurrence of metastable configurations and hysteresis at low temperatures in our simulations. The occurrence of phase separation in models with long-range electron-phonon coupling had been sug- 

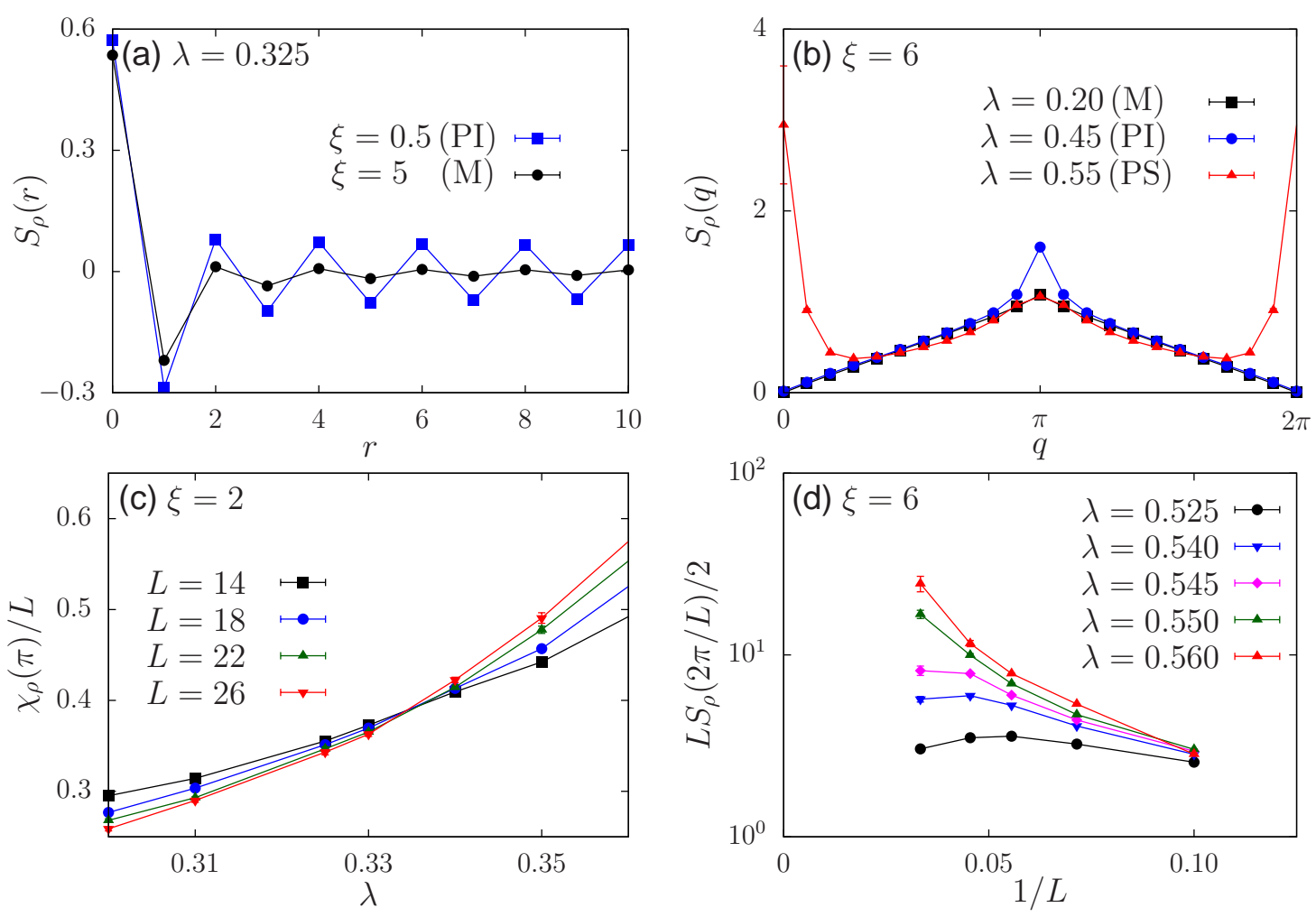

FIG. 2. (Color online) (a) Density-density correlation function $S_{\rho}(r)$, for $\lambda=0.325$ and $\beta t=L=22$, in the Peierls insulator (PI, $\xi=0.5)$ and the metallic phase $(\mathrm{M}, \xi=5)$. (b) Structure factor $S_{\rho}(q)$ at $\xi=6$ in the three phases of Fig. 1 . We used $L=22$, and $\beta=L(L / 2)$ for $\lambda=0.2,0.45(0.55)$. (c) Scaling of the charge susceptibility $\chi_{\rho}(\pi)[$ Eq. (5)] at $\xi=2$, defining the critical point $\lambda_{\mathrm{c}}^{\mathrm{PI}}=0.33(1)$ of the Peierls transition. Here $\beta=L$. (d) Finite-size scaling of $\pi S_{\rho}\left(q_{1}\right) / q_{1}=L S_{\rho}(2 \pi / L) / 2$ with $\beta=L / 2$. The divergence for $\lambda \geq 0.55$ indicates phase separation (PS). All results are for $\omega_{0} / t=0.5$.

gested before [20] and observed in analytical work [6, 7]; for short-range interactions, it is suppressed by the absence of bound triplet states 20, but may occur in the vicinity of a Mott transition [37. Phase separation is expected to be suppressed in the presence of additional long-range electron-electron interaction 38.

In combination with analytical continuation [39, we can calculate the single-particle spectral function

$A(k, \omega)=\frac{1}{Z} \sum_{i j}\left|\left\langle i\left|c_{k \sigma}\right| j\right\rangle\right|^{2}\left(e^{-\beta E_{i}}+e^{-\beta E_{j}}\right) \delta\left(\Delta_{j i}-\omega\right)$,

where $|i\rangle$ is an eigenstate with energy $E_{i}$, and $\Delta_{j i}=$ $E_{j}-E_{i}$. Results in the metallic phase $(\lambda=0.2)$ are shown in Fig. 3 for the extreme Holstein $(\xi=0.1)$ and Fröhlich limits $(\xi=\infty)$. The locus of spectral weight follows the free band dispersion, $-2 t \cos k$. The exponentially small Luther-Emery spin gap is not resolved for the parameters chosen, and the spectrum is particle-hole symmetric. Excitations are sharp inside the coherent interval $\left[-\omega_{0}, \omega_{0}\right]$, whereas they are substantially broadened as a result of multiphonon processes at higher energies [40]. As expected for our 1D model, the spectrum agrees well with the exact bosonization result [41, which predicts a hybridization of the spin, charge and phonon modes, although spin-charge separation is not visible due to the weak coupling and small $\omega_{0}$ 42. Comparing Figs. 3(a) and (b) we see that in contrast to previous work on one and two electrons $[8,10,19,22$, the impact of the interaction range is remarkably small. This important characteristic of the many-electron case can be related to the absence of significant polaron and bipolaron effects in the metallic phase of Fig. 1 .

Figures $4(\mathrm{a}),(\mathrm{b})$ show $A(k, \omega)$ in the Peierls phase $(\lambda=0.4)$. In the Holstein regime $(\xi=0.1)$, the spectrum consists of two sets of features. The cosine band seen in Fig. 3 has acquired a gap at the Fermi level and reveals additional, backfolded shadow bands as a result of dimerization and the corresponding doubling of the unit cell [43]. These signatures are labeled (1) and (1') in Fig. 4(a), respectively. In addition, we find lower-energy excitations labeled (2) corresponding to bound solitonantisoliton pairs or, equivalently, polarons [30, 44, which are absent in a homogeneous mean-field solution that captures only (1) and (1') [30. The soliton dispersion indicates a mass larger than the electron mass. Because their energy at the Fermi level is lower than the Peierls gap, doping would lead to the formation of solitons 45.

Increasing the interaction range from $\xi=0.1$ to $\xi=4$ 


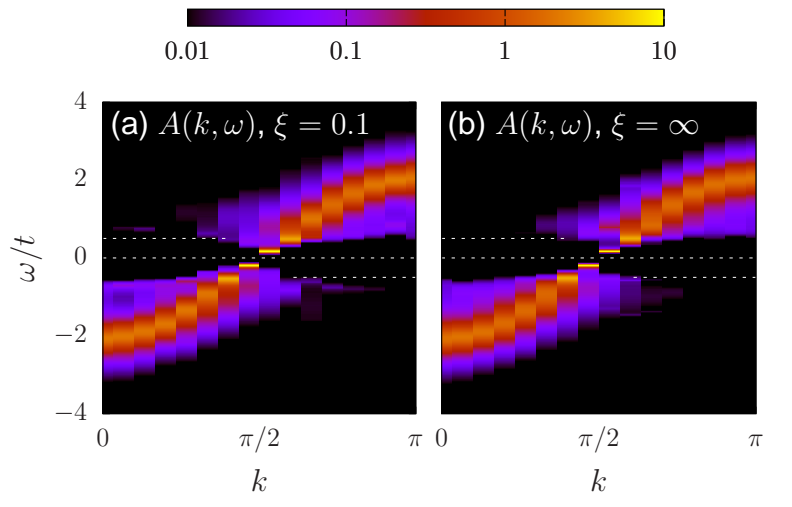

FIG. 3. (Color online) Single-particle spectral function $A(k, \omega)$ [Eq. [6] ] in the metallic phase at $\lambda=0.2$ for (a) $\xi=0.1$, (b) $\xi=\infty$. Here $\omega_{0} / t=0.5, \beta t=L=30$. The dashed lines indicate the Fermi level $(\omega=0)$ and $\omega= \pm \omega_{0}$.
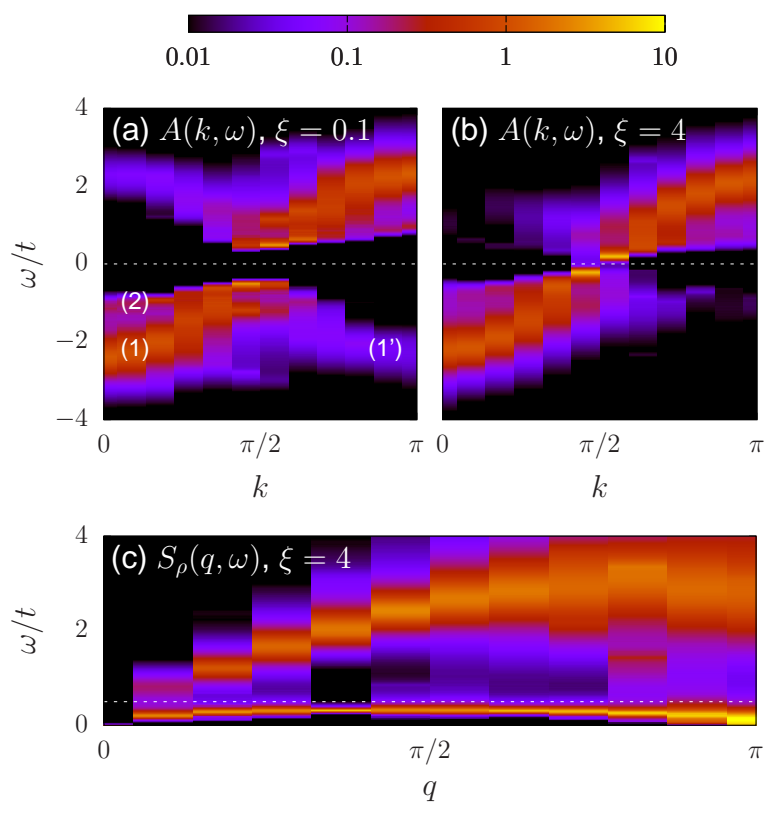

FIG. 4. (Color online) (a),(b) Single-particle spectral function $A(k, \omega)$ in the Peierls phase at $\lambda=0.4$ for (a) $\xi=0.1$, (b) $\xi=4$. (c) Dynamical charge structure factor [Eq. (7)] for the same parameters as in (b). Here $\omega_{0} / t=0.5, \beta t=L=22$. The dashed lines indicate (a),(b) the Fermi level and (c) $\omega_{0}$. The labels (1), (1'), and (2) in (a) are explained in the text.

drives the system into the vicinity of the metal-insulator transition, see Fig. 1. This is reflected by a much smaller gap at the Fermi level, reduced spectral weight of the polaron excitations, and the suppression of shadow bands. Consequently, the spectral function becomes quite similar (but not identical) to that shown in Fig. 3 , and illustrates the continuous evolution of $A(k, \omega)$ across $\lambda_{\mathrm{c}}^{\mathrm{PI}}$.

A hallmark feature of the Peierls state is phonon softening at $q=2 k_{\mathrm{F}}$, which is visible [30, 46] in the dynami-
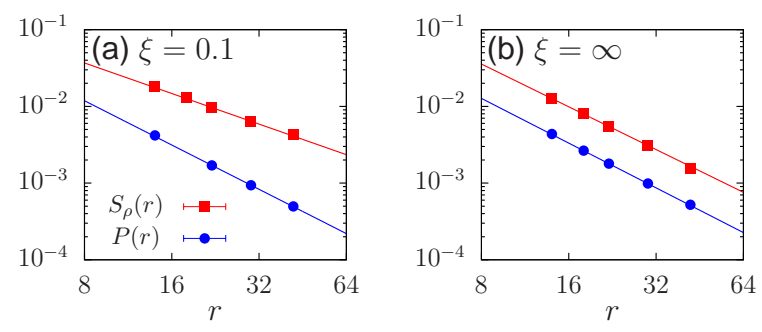

FIG. 5. (Color online) Charge and pairing correlations at distance $r=L / 2$ for (a) $\xi=0.1$, (b) $\xi=\infty$. Here $\omega_{0} / t=0.5$ and $\lambda=0.2$. Lines are fits to a power law $f(r)=A r^{-\eta}$.

cal charge structure factor $\left[\hat{\rho}_{q}=\sum_{r} e^{i q r}\left(\hat{n}_{r}-\left\langle\hat{n}_{r}\right\rangle\right) / \sqrt{L}\right]$

$$
S_{\rho}(q, \omega)=\frac{1}{Z} \sum_{i j}\left|\left\langle i\left|\hat{\rho}_{q}\right| j\right\rangle\right|^{2} e^{-\beta E_{j}} \delta\left(\Delta_{j i}-\omega\right)
$$

The results in Fig. 4(c) for $\lambda=0.4, \xi=4$ [the same parameters as in Fig. 4(b)] reveal a clear signature of the renormalized phonon dispersion. The spectrum is dominated by the soft phonon mode at $\omega=0, q=2 k_{\mathrm{F}}$. Furthermore, we observe a continuum of particle-hole excitations, and a charge gap at long wavelengths $(q \rightarrow 0)$.

Finally, we consider the interaction-range effect on the competition of charge and pairing correlations. Figure 5 shows results for $S_{\rho}(r)$ and the s-wave pair correlator $P(r)=\left\langle\Delta_{r}^{\dagger} \Delta_{0}\right\rangle$ (with $\Delta_{r}^{\dagger}=c_{r \uparrow}^{\dagger} c_{r \downarrow}^{\dagger}$ ) in the metallic phase. For $\xi=0.1$, Fig. 5(a) reflects the dominance of charge correlations previously observed for the Holstein model [47. However, Fig. 5(b) reveals that long-range electron-phonon interaction (here $\xi=\infty$ ) suppresses charge correlations and thereby results in almost identical power-law exponents in both channels. Additional shortrange electron-electron repulsion is expected to further suppress onsite bipolaron formation-promoting nonlocal pairing - and to lead to a more general phase diagram with Mott, metallic, Peierls and phase-separated ground states. It will be interesting to explore this issue further, both at and away from half filling, also in the light of a recently reported phase of correlated singlets [48].

Conclusions.-We used exact Monte Carlo simulations to study many-electron systems with nonlocal and even long-range electron-phonon interaction. Compared to the Holstein model, extended interactions suppress the Peierls instability - making pairing more favorable - and can lead to phase separation. Key implications for materials modeling are that interactions of finite but small range are well described by Holstein-type models, whereas long-range interactions can have substantial effects on the balance of pairing and charge correlations.

We are grateful to F. Essler and A. Muramatsu for helpful discussions, and acknowledge support from the DFG Grant No. Ho 4489/2-1 as well as computer time at the LRZ Munich and the Jülich Supercomputing Centre. 
[1] Polarons in Advanced Materials, edited by A. S. Alexandrov (Canopus Publishing and Springer Verlag $\mathrm{GmbH}$, Bristol (UK), 2007).

[2] T. Holstein, Ann. Phys. (N.Y.) 8, 325; 8, 343 (1959).

[3] W. Barford, Electronic and Optical Properties of Conjugated Polymers (Oxford University Press, Oxford, 2005).

[4] H. Fröhlich, Adv. Phys. 3, 325 (1954).

[5] R. Zeyher, Z. Phys. B 80, 187 (1990).

[6] A. S. Alexandrov and P. E. Kornilovitch, J. Phys.: Condens. Matter 14, 5337 (2002).

[7] A. S. Alexandrov and P. E. Kornilovitch, Phys. Lett. A 299, 650 (2002).

[8] J. T. Devreese and A. S. Alexandrov, Rep. Prog. Phys. 72, 066501 (2009).

[9] T. M. Hardy, J. P. Hague, J. H. Samson, and A. S. Alexandrov, Phys. Rev. B 79, 212501 (2009).

[10] H. Fehske, J. Loos, and G. Wellein, Phys. Rev. B 61, 8016 (2000).

[11] J. Bonča, T. Katrašnik, and S. A. Trugman, Phys. Rev. Lett. 84, 3153 (2000).

[12] J. P. Hague, P. E. Kornilovitch, J. H. Samson, and A. S. Alexandrov, Phys. Rev. Lett. 98, 037002 (2007).

[13] R. Blankenbecler, D. J. Scalapino, and R. L. Sugar, Phys. Rev. D 24, 2278 (1981).

[14] J. E. Hirsch and E. Fradkin, Phys. Rev. Lett. 49, 402 (1982).

[15] H. Fehske, G. Hager, and E. Jeckelmann, Europhys. Lett. 84, 57001 (2008).

[16] H. Fehske, G. Wellein, G. Hager, A. Weiße, and A. R. Bishop, Phys. Rev. B 69, 165115 (2004).

[17] R. T. Clay and R. P. Hardikar, Phys. Rev. Lett. 95, 096401 (2005).

[18] F. F. Assaad and T. C. Lang, Phys. Rev. B 76, 035116 (2007).

[19] P. E. Kornilovitch, Phys. Rev. Lett. 81, 5382 (1998).

[20] J. Bonča and S. A. Trugman, Phys. Rev. B 64, 094507 (2001).

[21] A. S. Mishchenko, N. V. Prokof'ev, A. Sakamoto, and B. V. Svistunov, Phys. Rev. B 62, 6317 (2000).

[22] A. S. Mishchenko, N. Nagaosa, N. V. Prokof'ev, A. Sakamoto, and B. V. Svistunov, Phys. Rev. Lett. 91, 236401 (2003).

[23] R. Peierls, Surprises in Theoretical Physics (Princeton University Press, New Jersey, 1979).
[24] D. Jérome, Chem. Rev. 104, 5565 (2004).

[25] E. Jeckelmann, C. Zhang, and S. R. White, Phys. Rev. B 60, 7950 (1999).

[26] A. N. Rubtsov, V. V. Savkin, and A. I. Lichtenstein, Phys. Rev. B 72, 035122 (2005).

[27] A. S. Alexandrov and P. E. Kornilovitch, Phys. Rev. Lett. 82, 807 (1999).

[28] R. P. Feynman, Phys. Rev. 97, 660 (1955).

[29] P. Werner and A. J. Millis, Phys. Rev. Lett. 99, 146404 (2007).

[30] M. Hohenadler, H. Fehske, and F. F. Assaad, Phys. Rev. B 83, 115105 (2011).

[31] M. Hohenadler, D. Neuber, W. von der Linden, G. Wellein, J. Loos, and H. Fehske, Phys. Rev. B 71, 245111 (2005).

[32] R. P. Hardikar and R. T. Clay, Phys. Rev. B 75, 245103 (2007).

[33] M. Tezuka, R. Arita, and H. Aoki, Phys. Rev. B 76, 155114 (2007).

[34] J. Voit, Rep. Prog. Phys. 58, 977 (1995).

[35] S. Ejima and H. Fehske, J. Phys. Conf. Ser. 200, 012031 (2010).

[36] J. E. Hirsch and E. Fradkin, Phys. Rev. B 27, 4302 (1983).

[37] M. Capone, G. Sangiovanni, C. Castellani, C. Di Castro, and M. Grilli, Phys. Rev. Lett. 92, 106401 (2004).

[38] A. S. Alexandrov and V. V. Kabanov, Pis'ma Zh. Eksp. Teor. Fiz. 72, 825 (2000), [JETP Lett. 72, 569 (2000)].

[39] K. S. D. Beach, cond-mat/0403055.

[40] J. Loos, M. Hohenadler, and H. Fehske, J. Phys.: Condens. Matter 18, 2453 (2006).

[41] V. Meden, K. Schönhammer, and O. Gunnarsson, Phys. Rev. B 50, 11179 (1994).

[42] W. Q. Ning, H. Zhao, C. Q. Wu, and H. Q. Lin, Phys. Rev. Lett. 96, 156402 (2006).

[43] J. Voit, L. Perfetti, F. Zwick, H. Berger, G. Margaritondo, G. Grüner, H. Höchst, and M. Grioni, Science 290, 501 (2000).

[44] A. J. Heeger, S. Kivelson, J. R. Schrieffer, and W. P. Su, Rev. Mod. Phys. 60, 781 (1988).

[45] W. P. Su, J. R. Schrieffer, and A. J. Heeger, Phys. Rev. Lett. 42, 1698 (1979).

[46] F. F. Assaad, Phys. Rev. B 78, 155124 (2008).

[47] K.-M. Tam, S.-W. Tsai, and D. K. Campbell, Phys. Rev. B 84, 165123 (2011).

[48] S. Reja, S. Yarlagadda, and P. B. Littlewood, Phys. Rev. B 86, 045110 (2012). 